\title{
Novel design for transparent high-pressure fuel injector nozzles
}

\author{
Z. Falgout ${ }^{1, a)}$ and M. Linne ${ }^{1,2}$ \\ ${ }^{1}$ Department of Applied Mechanics, Chalmers University, 41296 Gothenburg, Sweden \\ ${ }^{2}$ School of Engineering, The University of Edinburgh, EH8 3JL Edinburgh, United Kingdom
}

(Received 27 April 2016; accepted 23 July 2016; published online 9 August 2016)

\begin{abstract}
The efficiency and emissions of internal combustion (IC) engines are closely tied to the formation of the combustible air-fuel mixture. Direct-injection engines have become more common due to their increased practical flexibility and efficiency, and sprays dominate mixture formation in these engines. Spray formation, or rather the transition from a cylindrical liquid jet to a field of isolated droplets, is not completely understood. However, it is known that nozzle orifice flow and cavitation have an important effect on the formation of fuel injector sprays, even if the exact details of this effect remain unknown. A number of studies in recent years have used injectors with optically transparent nozzles (OTN) to allow observation of the nozzle orifice flow. Our goal in this work is to design various OTN concepts that mimic the flow inside commercial injector nozzles, at realistic fuel pressures, and yet still allow access to the very near nozzle region of the spray so that interior flow structure can be correlated with primary breakup dynamics. This goal has not been achieved until now because interior structures can be very complex, and the most appropriate optical materials are brittle and easily fractured by realistic fuel pressures. An OTN design that achieves realistic injection pressures and grants visual access to the interior flow and spray formation will be explained in detail. The design uses an acrylic nozzle, which is ideal for imaging the interior flow. This nozzle is supported from the outside with sapphire clamps, which reduces tensile stresses in the nozzle and increases the nozzle's injection pressure capacity. An ensemble of nozzles were mechanically tested to prove this design concept. Published by AIP Publishing. [http://dx.doi.org/10.1063/1.4960402]
\end{abstract}

\section{INTRODUCTION}

\section{A. Motivation}

In modern direct-injection engines, elevated injection pressures and injectors with microscopic nozzle orifices are used to enhance atomization and deliver sufficient fuel mass quickly. The spray produced by these injectors is both very complex and very important for subsequent mixture formation and combustion, and the effect of geometric details of the injector on the spray is difficult to study experimentally. The injector interior flow has a dominant effect on spray formation, and so both interior flow and spray formation dynamics should be captured simultaneously. The flow inside most injectors must turn abruptly to enter the nozzle orifices, which in many cases causes cavitation to occur in the flow. Cavitation adds complexity to the interior flow and thereby also spray formation, and while some forms of cavitation have been shown to be beneficial for atomization, hydraulic flip suppresses atomization. ${ }^{1}$ Until cavitation is better understood and easier to control, it is usually avoided, if possible. The two most common ways to reduce orifice flow cavitation are hydrogrinding ${ }^{2}$ and tapering the orifice.

Hydraulic characterization is often used to examine the extent of cavitation in the nozzle orifice of commercially available injectors, ${ }^{3-5}$ together with the global flow rates inside the orifice. The information provided by this measurement is

\footnotetext{
a) Author to whom correspondence should be addressed. Electronic mail: falgout@chalmers.se
}

limited, and it is possible that cavitation may still exist even when hydraulic characterization measurements indicate that it is unlikely. This method disrupts the spray, and is therefore not able to characterize the effect of interior flow on spray formation. Another method to study cavitation in real injectors is to use $\mathrm{x}$-rays to probe the interior flow field. Phase contrast imaging (PCI) has been used recently to capture cavitation in an unmodified commercially available injector. ${ }^{6}$ Cerium was added to the fuel to enhance image contrast. The cavitation's static location was extracted in some cases, while in others it was still unclear whether or not cavitation was present, perhaps due to persisting low image contrast.

An alternative way to study interior flow is to construct an optically transparent nozzle (OTN) with the same interior geometry as the real injector nozzle. This allows features such as cavitation to be imaged, and spatially resolved velocity fields to be captured with techniques like particle image velocimetry (PIV or micro-PIV). ${ }^{7}$ There is a limit to the orifice size that can be used with PIV, however. An important point is that the refractive indices of the nozzle material and fuel must match, otherwise the orifice edges will appear dark in back-lit images, which obscures the areas where cavitation is likely to occur. Unfortunately, this requirement presents some challenges. Widely available optically transparent engineering materials are often brittle at room temperature. While brittle materials often have higher theoretical strengths, microscopic flaws left from processing can significantly reduce the finished nozzle's load capacity. Acrylic and quartz have an index that comes close to many fuels, but unfortunately they usually break well before realistic fuel pressures are reached. Acrylic 
is usually stronger than quartz because of its tendency for limited plastic deformation before failure, which reduces the probability of failure caused by microscopic stress concentrating flaws by allowing them to deform locally in response to elevated stress instead of growing catastrophically. Ceramics such as quartz tend to display almost no plastic deformation before failure. Sapphire, on the other hand, has strength properties similar to steel, but it is poorly index matched to the fluids of interest.

The vast majority of the studies that have used OTN to study interior flow of fuel injector nozzles use injection pressures significantly lower than the corresponding commercially available injector, due to the challenge of designing an OTN which can withstand high injection pressures. To compensate, some used scaled-up geometries to match the Reynolds number, although there are several aspects of the flow that do not scale equally. ${ }^{6}$ Studies at realistic injection pressures and scales are more likely to gain knowledge relevant to the real flow of interest. All of the designs that had realsized interior flow passages were so large that primary breakup (spray formation) could not be studied, which is unfortunate since spray formation and interior flow are very closely linked.

\section{B. Past studies}

Arcoumanis et al. examined the effect of scaling geometries while matching Reynolds and cavitation numbers in the real-size and a scaled up geometry. ${ }^{8}$ They found that the cavitation structures were not identical between the two types of nozzles, but there were enough qualitative similarities for the flows to be understood. Their injectors were made of acrylic glass, and were attached to a Bosch common-rail injector body. The acrylic OTN's overall size was several times larger than injector body, and so spray formation was not accessible with the design.

Reid et al. used sapphire plates to create a simplified version of the geometry found in valve-covered-orifice injectors. ${ }^{9,10}$ They achieved injection pressures of 2000 bars with the design. The orifice was constructed by stacking sapphire plates with holes of various sizes on top of each other. These holes formed the desired flow geometry. The device was sealed by shear force, and the plates were held in place with a large metal fixture which hid the spray formation region from view. The refractive index mismatch made orifice flow visualization impossible. This was not an issue in the sac volume because the sac volume had a much larger radius. The small nozzlefuel interface curvature normal to the line of sight reduced the effect of the refractive index mismatch.

Badock et al. achieved elevated injection pressures using acrylic nozzles, although much like Arcoumanis et al., the piece was significantly larger than the original injector nozzle. ${ }^{11}$ Studying primary breakup of a spray in such a nozzle is impossible, because the surface of the drilled holes that limit the orifice length distorts spray images. This is because the nozzle material and air have different refractive indices.

Blessing et al. achieved injection pressures of 800 bars using an acrylic injector nozzle with a single off-axis orifice. ${ }^{2}$ The design was compact enough to allow spray formation to be studied simultaneously with the in-nozzle orifice cavitation.
Unfortunately, no further information regarding the design, such as dimensions or sealing mechanism, was provided. The orifice hole was significantly smaller than the injector in the current study, which is indicated by the analyses in this study to reduce the stress in the nozzle significantly and might explain why they were able to achieve these injection pressures with only acrylic.

Mitroglou et al. ${ }^{12}$ achieved 400 bars injection pressure with an acrylic OTN design that identically matched the interior geometry of a six-orifice heavy duty diesel road vehicle engine. The acrylic piece was attached to a commercially available injector with the nozzle machined away by pressing the acrylic nozzle against the injector nose with a lever mechanism. The quality of the interior surfaces was examined optically and the nozzles were discarded after 40 injections to prevent the degradation of the nozzle from affecting the recorded flows. Hundreds of nozzles were used to capture the ensemble and average cavitation images. An o-ring was used to seal the interface between the acrylic nozzle and the metal injector body.

Hayashi et al. ${ }^{13}$ reached 500 bars with an OTN for a realsized light-duty diesel injector. The nozzle was made from quartz, and so was able to sustain elevated temperatures. The effects of geometric and string cavitation on spray formation, ignition delay, and flame width were compared. The details of the assembly were not included in the paper.

Butcher et al. ${ }^{14}$ constructed a quartz nozzle in order to study flash boiling sprays relevant to spark-ignition engines and even installed a modified needle to reduce the stress concentration in the nozzle from the needle seat sealing force. Their nozzle was made of quartz because they wanted to investigate the flows at elevated ambient temperatures. The orifice hole in their nozzle was made with a diamond core drill, and efforts were made to minimize the damage at the orifice inlet and exit caused by this process. The group even used scanning-electron microscopy to study the geometry of silicone nozzle molds and examine the ability of hydrogrinding to reduce this damage. Ultimately, their injection pressures were limited to 40 bars.

In summary, the three most common materials used in optically transparent injector nozzle designs are acrylic glass, sapphire, and quartz (fused silica). All of the past studies constructed the nozzle out of a single monolithic piece of one of these materials. There are more studies which successfully reach elevated pressures with acrylic than the other materials, although none of these studies reached the operating pressures of the real injector. In most of these studies, the body of the OTN was much larger than the corresponding metal injector nozzle, which made it impossible to study the spray formation region and the orifice flow simultaneously. This might have been necessary to reach elevated injection pressures with these designs.

\section{Predicting failure of optically transparent materials}

The most common optically transparent materials used in past studies are brittle at room temperature, where most studies of cavitation in diesel injectors are conducted. Brittle materials usually fail over a wide variety of loads in practical testing, and 
so statistical descriptions of their failure tend to be more accurate. The most common probability distribution used for brittle material failure stress is the Weibull distribution. The failure probability of a given finished workpiece due to processing flaws may be expressed as a function of the size distribution of the flaws that left in the material's surface, summarized by $\sigma_{p}$ and $k$, material parameters characterizing sub-critical crack growth, $N$ and $B$, and the distribution of stress in the design during operation as a function of the number of loading cycles, $n$, is $^{15}$

$$
P_{f}(n)=1-\exp \left(-\sum_{i} V_{i} \sigma_{p}^{-k}\left(\frac{\sigma_{i}^{N} n}{B}\right)^{\frac{k}{N-2}}\right)
$$

where $\sigma_{i}$ is the maximum tensile stress in a given finite element with a volume of $V_{i}$. This formulation neglects the relative orientation of the flaws and the principal stresses, as well as the effect of the principal stresses other than the largest one. This formulation can be simplified under the assumption of a steady stress field while under load and uniform material parameters

$$
\begin{gathered}
P_{f}=1-\exp \left(-C_{1} n^{C_{2}}\right), \\
C_{1}=\sum_{i} V_{i} \sigma_{i}^{N C_{2}} \sigma_{p}^{-k} B^{-C_{2}}=C_{3} \sum_{i} V_{i} \sigma_{i}^{N C_{2}}, \\
C_{2}=\frac{k}{N-2}, \\
C_{3}=\sigma_{p}^{-k} B^{-C_{2}} .
\end{gathered}
$$

For a finished material, the failure probability distribution is found empirically by testing several pieces to failure ${ }^{16-18}$ in order to obtain the constants in Equation (1). Such test data are not available for the optically transparent materials used in past studies, and even if they were, predicting the failure probability of these materials accurately is more complicated than simply reusing these data when the stress on the material is similar. The constants in these equations are valid for a given shaping process, and so they are valid usually only for the finished design which was tested to obtain them, or for pieces that have been processed in a similar way. This is because processing leaves brittle materials with microscopic cracks that become stress concentrations during operation. The properties of these cracks are difficult to predict, because the factors affecting their size and shape are not carefully controlled in practical processing techniques.

Measures can be taken to reduce the extent of the flaws at the finished piece's surface and thereby reduce failure probability. In the case of brittle ceramics, processing the material more slowly is less likely to produce large cracks that cause failure at lower stresses. ${ }^{16}$ For brittle polymers, a processing speed that balances cutting force and thermal degradation due to frictional forces should be used. After manufacturing, annealing in a thermal ${ }^{19}$ or chemical ${ }^{20}$ bath has also been shown to repair flaws.

\section{General trends in OTN mechanical behavior}

In order to investigate the mechanical behavior of OTN in general, a simplified geometry with few degrees of freedom was investigated over a range of geometries and load condi-

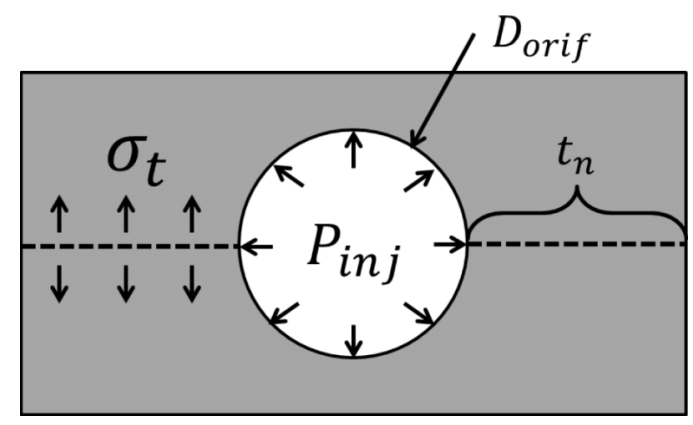

FIG. 1. Geometry for simplified injector nozzle mechanical model.

tions. This simplified model consists of a rectangular section with a circular void in the center to represent an interior flow passage shown in Figure 1. A rectangular outer profile must be used for optically transparent flow devices because a curved outer surface distorts images of the interior flow. This model can also be used as an analogy for many other transparent flow rigs.

Assuming that the geometry is rigid, if a uniform pressure, $P_{i n j}$, is applied to the boundary of the circular orifice with a diameter of $D_{\text {orif }}$, then the tensile stress at any point along a line normal to the inner boundary scales as

$$
\sigma_{t} \propto \frac{P_{i n j} D_{o r i f}}{t_{n}},
$$

where $t_{n}$ represents the thickness of the piece along this line. While $\sigma_{t, \max }$ is not as complete a metric as failure probability, the two are correlated. Given a fixed geometry and injection pressure, the maximum stress occurs where the piece is thinnest, or where $t_{n}=t_{n, \text { min }}$. This model is more realistic if elasticity is added. Since no analytical solution exists for the stress field in an elastic material with the geometry and load shown in Figure 1, a commercial finite element analysis (FEA) software, ANSYS Workbench 14.5, was used to calculate the effect of variations in an elastic model geometry on the stress field. The numerical domain is shown in Figure 2, with symmetry conditions imposed along the dashed boundaries and pressure applied as shown.

The parameters shown in Fig. 2 were varied independently over the ranges shown in Table I to examine the behavior of the maximum stress. The stress field from a representative case is shown in Fig. 3. The maximum stress showed dependence only on the minimum of $t_{1}$ and $t_{2}$, and so these parameters were replaced by the minimum of the two, $t_{n, \text { min }}$. Also, the

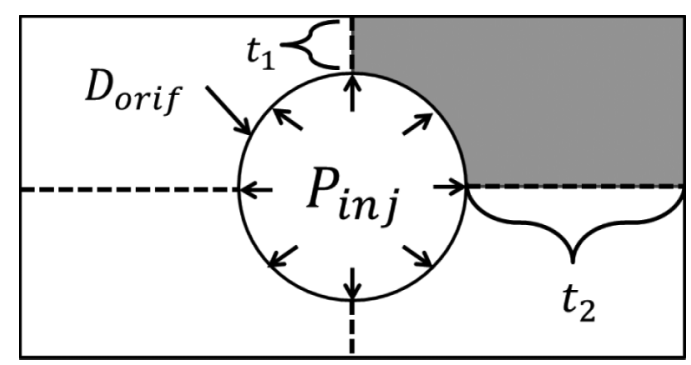

FIG. 2. Simplified model with numerical domain for parameter sweeps colored in gray, with lines of symmetry and parameters labeled. 
TABLE I. Parameter ranges used to fit Eq. (5).

\begin{tabular}{lccc}
\hline \hline Parameter & Min & Max & Unit \\
\hline$P_{\text {inj }}$ & 20 & 80 & MPa \\
$D_{\text {orif }}$ & 0.375 & 3 & $\mathrm{~mm}$ \\
$t_{n, \text { min }}$ & 0.375 & 1.5 & $\mathrm{~mm}$ \\
\hline \hline
\end{tabular}

independent variations of $D_{\text {orif }}$ and $t_{n, \min }$ produced identical maximum stress at values where their ratio $D_{\text {orif }} / t_{n, \min }$ coincided. $D_{\text {orif }}$ and $t_{n, \min }$ were replaced by their ratio in the parameter set as a result. The dependence of the maximum stress on pressure in the resulting dataset was clearly linear, while the dependence of the maximum stress on $D_{\text {orif }} / t_{n, \min }$ was slightly exponential. Linear regression was used to find the exponent of $D_{\text {orif }} / t_{n, \min }$ producing the following most probable relation for the maximum stress:

$$
\sigma_{t, \max }=P_{i n j}\left(\frac{D_{\text {orif }}}{t_{n, \min }}\right)^{0.643} .
$$

In order to calculate the failure probability, real data are needed to calculate the constants in Equations (2) and (3). However, the maximum stress in the piece can be used to compare cases and often does correlate with failure probability (it appears in the sum on the RHS of Equation (1)). Also, while the nozzle geometry is always more complicated than the geometry of this simplified model, this model does indicate important trends. Transparent nozzles with larger orifices will have larger internal stresses. The stress in the nozzle piece will also be larger in regions where the nozzle is thin. The spray formation region is only visible if the nozzle thickness is not larger than the length of the orifice in the direction of the orifice central axis, so this establishes a trade-off between spray flow access and mechanical performance.

The thickness for the final geometries is unknown for all of the reviewed past studies, but the force on the nozzle cross section can be calculated. This is shown in Table II for the past studies listed to allow a comparison of the relative performance of their designs. The possibility to visualize spray formation is also included in Table II, since including this feature limits the thickness of the nozzle. The design of Blessing et al. came closest to the operating condition of a real injector while providing optical access to both the interior and spray formation flows, however, none of the nozzles achieved greater than $50 \%$ of realistic injection pressures while granting visual access to both the spray and interior flows.
$1,4 \mathrm{e} 8 \mathrm{Max}$

$1,2 \mathrm{e} 8$

$1,1 \mathrm{e} 8$

$9,3 e 7$

$7,8 \mathrm{e} 7$

$6,2 e 7$

$4,6 \mathrm{e} 7$

$3,1 \mathrm{e} 7$

$1,5 \mathrm{e} 7$

$-1,3 e 5$ Min

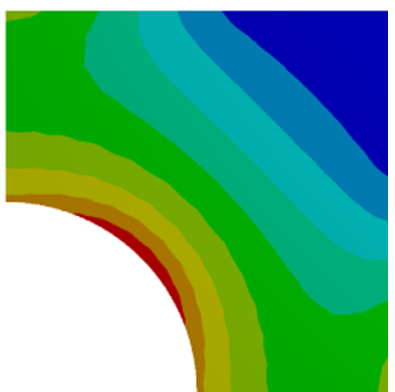

FIG. 3. Maximum tensile stress (Pa) field from the case where $D_{\text {orif }} / t_{n, \min }=$ 2 and $P_{i n j}=80 \mathrm{MPa}$.

\section{DESIGN DETAILS}

Acrylic has been shown historically to be more durable than quartz while having nearly an ideal refractive index, and so it was chosen as the material for the OTN section. Acrylic is also cheaper and easier to process than either quartz or sapphire. Index-matching the nozzle material with the fuel becomes more important for effectively imaging the flow inside the orifices as they become smaller due to the increasing curvature of the fluid-solid interface.

For this study, the injector geometry of interest belongs to a heavy-duty marine diesel engine fuel injector that normally operates with 800 bars injection pressure. The geometry of the experimental nozzle used in the current study is halfscale for reasons that do not involve its mechanical durability. A design cross-section illustration that shows the experimental injector's internal geometry is presented in Figure 4. The commercial injector uses a stop shaft rather than a needle valve to control the fuel supply to the injector orifices. The stop shaft is stationary in this design, and it was brazed onto the nozzle a commercial SCANIA XP injector that supplied fuel flow. The experimental nozzle assembly joins to the stop shaft using set screws. All fluid seals between pieces in the assembly are made with 90A hardness o-rings. An important design feature is the pressure tap that feeds directly into the sac volume, which is called out in Figure 4. This allows timeresolved injection pressures to be captured for each injection, confirming that the o-ring seals are holding and that the injection pressure is near the set pressure specified with the fuel supply system.

Rigid clamps with considerable thickness were added to the assembly to support the transparent acrylic nozzle piece from the outside and limit its expansion. Sapphire was chosen

TABLE II. Past studies using OTN at elevated injection pressures.

\begin{tabular}{lcccccc}
\hline \hline \multicolumn{1}{c}{$\begin{array}{c}P_{\text {inj }} \\
\text { Study }\end{array}$} & & & & & \\
(bars) & $D_{\text {orif }}(\mathrm{mm})$ & Load $(\mathrm{kN})$ & Material & Orifice flow & Spray formation \\
Arcoumanis et al. & 332 & 0.176 & 5.84 & Acrylic & $\mathrm{Y}$ & $\mathrm{N}$ \\
Reid et al. & 2050 & 0.3 & 61.5 & Sapphire & $\mathrm{N}$ & $\mathrm{N}$ \\
Blessing et al. & 800 & 0.2 & 16 & Acrylic & $\mathrm{Y}$ & $\mathrm{Y}$ \\
Badock et al. & 250 & 0.2 & 5 & Acrylic & $\mathrm{Y}$ & $\mathrm{N}$ \\
Butcher et al. & 40 & 0.2 & 0.8 & Quartz & $\mathrm{Y}$ & $\mathrm{Y}$ \\
Mitroglou et al. & 400 & 0.16 & 6.4 & Acrylic & $\mathrm{Y}$ & $\mathrm{Y}$ \\
Hayashi et al. & 500 & 0.14 & 7 & Quartz & $\mathrm{Y}$ & $\mathrm{Y}$ \\
\hline \hline
\end{tabular}




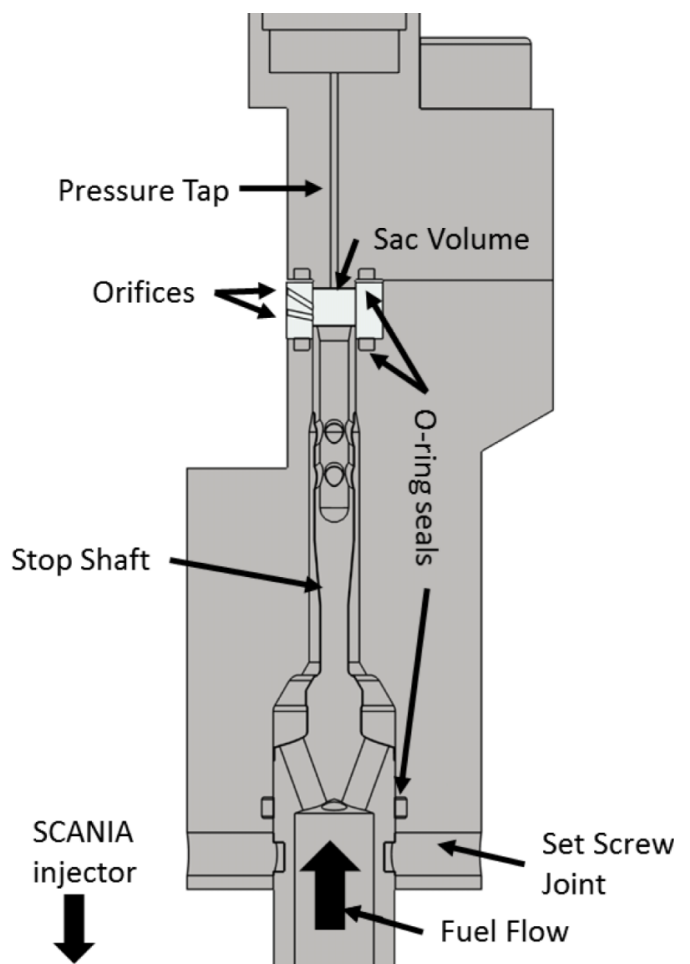

FIG. 4. Illustration of design cross section with internal features called out.

for the construction of these clamps due to its high durability and transparency. This combined the mechanical strength of sapphire with the advantages of acrylic for the nozzle piece for imaging. The acrylic nozzle piece protruded from the rest of the assembly slightly so that the sapphire clamps would make contact with only the acrylic piece. Drilling clearance holes for clamping bolts in a sapphire piece would significantly weaken the piece, so instead the clearance holes for the clamping bolts were drilled through metal pieces that held rectangular sapphire windows. The edges of the windows were chamfered to avoid stress concentrations. These sapphire windows had flat, parallel exterior surfaces to minimize their effect on imaging. An illustration of the final design assembled with the metal clamps and sapphire inserts is shown in Figure 5(a). The design without metal clamps or sapphire is shown on the right side of Figure 5(b) for comparison. In order to allow space for the threaded holes in the other components, the nozzle is much thicker in the direction corresponding to the viewing direction in Figure 4. The acrylic nozzle piece is thin in the other orthogonal directions to allow imaging of spray formation. The transparent acrylic nozzle piece's interior and exterior dimensions are provided in the Appendix for reference (Fig. 17).

\section{TESTING}

To examine the real benefits of the clamped design concept, the experimental injector nozzle was mechanically tested with diesel fuel supplied by a commercial reciprocating piston pump that was driven by a large electric motor. 50 nozzles were tested at 400 bars without the metal clamps (case I) and 30 nozzles were tested at 600 bars with the clamps (case II). To create even contact between the clamps and the

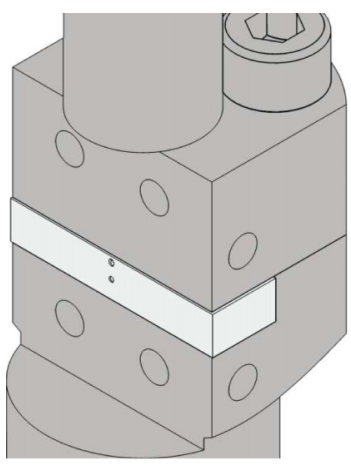

(a)

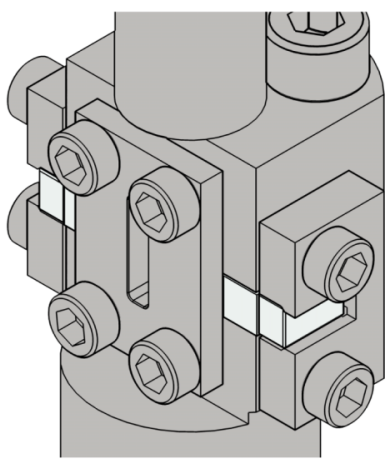

(b)
FIG. 5. (a) Illustration of design exterior without clamps (b) with clamps.

nozzle, the clamps were held flush against the surface of the nozzle before tightening the bolts by hand. When the nozzle failed, the sac volume pressure would drop suddenly before the commanded end of injection. The cumulative number of injections before failure was recorded for each nozzle.

Back-lit imaging was used to evaluate the visual access to interior cavitation and external spray flows granted by the design. The injection pressure was kept at 350 bars during visual testing to preserve the nozzles for mechanical testing. A Vision v1210 high speed camera with a long distance microscope, Questar model QM100, was used to capture high speed videos of the interior flow. The injector was illuminated from behind with a solid state plasma light source, Thor Labs $\mathrm{P} / \mathrm{N}$ HPLS243. The spray formation past the metal clamp was visible but out-of-focus, owing to the difference in the optical path lengths through the nozzle and through open air. Two separate imaging systems would be required to capture both flows simultaneously with optimal resolution. A simple illustration of the experimental setup is shown in Figure 6.

\section{A. Finite element analysis}

FEA was used to estimate the stresses in the finalized design to compare with the results from mechanical testing. The nozzle has a symmetry plane that includes the central axes of the two orifice holes, and this was used to reduce the computational domain in the FEA. Linear isotropic elasticities provided by the manufacturer were used for the materials in the model, which included the acrylic from the nozzle piece, the sapphire from the clamp inserts, and the steel of the other components. A sub-grid-scale contact model was used to prevent inter-penetration of the components, which had individual meshes. Pressure was applied to the interior faces of the acrylic nozzle: 400 bars to simulate case I and 600 bars for case II. The

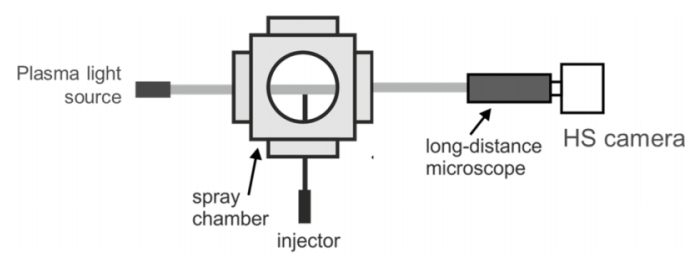

FIG. 6. Illustration of visual test rig. 
clamps were excluded from the simulation for case I. Because the clamping force during mechanical testing was unknown and applied by hand, it is assumed to be negligible, and therefore is not included in the simulations. Adaptive meshing was used to refine the numerical mesh until the peak stress in the piece changed by less than $5 \%$ between refinements.

\section{RESULTS}

The failure probability density and cumulative failure probability as a function of number of injections is shown in Fig. 7 for case I and Figure 8 for case II. There are outliers in the higher end of the failure distribution for case II, which are excluded from the cumulative failure probabilities for reasons that will be explained later. Fitting the experimental cumulative failure probabilities to Equation (2) yields values for the coefficients $C_{1}$ and $C_{2}$ of 0.002 and 1.7254 for case I and 0.0047 and 1.5305 for case II. The values for $C_{2}$, which, ideally should be constant for all tests of samples of the same finished design, are within $12 \%$ for the two cases. Assuming that $N \approx 1$, the FEA results can be used to provide an estimation for $C_{3}$ in the two tested cases by providing approximate values for the maximum tensile stress throughout the piece and the volumes of the associated finite elements in Equation (3). Using these data from the FEA simulations, excluding elements with maximum principal stresses that were compressive, and using the corresponding values of $C_{2}$ for the two cases produced estimations for $C_{3}$ of $4.6314 \times 10^{-9}$ and $2.6584 \times 10^{-7}$ for case I and case II, respectively. While, ideally, these constants should be the same for both loading cases, the parameters in the formulation of $C_{3}$ may, in fact, scale with local stresses. ${ }^{21}$ In that case, it seems logical to reuse these estimated parameters only for cases where the loads are similar in magnitude, which, according to Equation (5), scales strongly with injection pressure given a constant geometry.

\section{Case I}
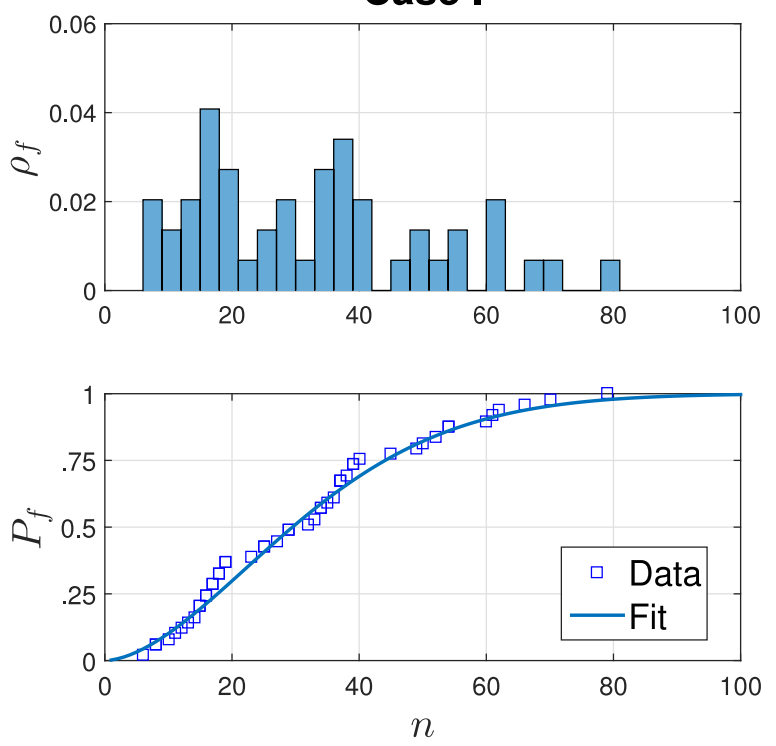

FIG. 7. Failure probability density distribution (above) and failure probability distribution (below) for case I.
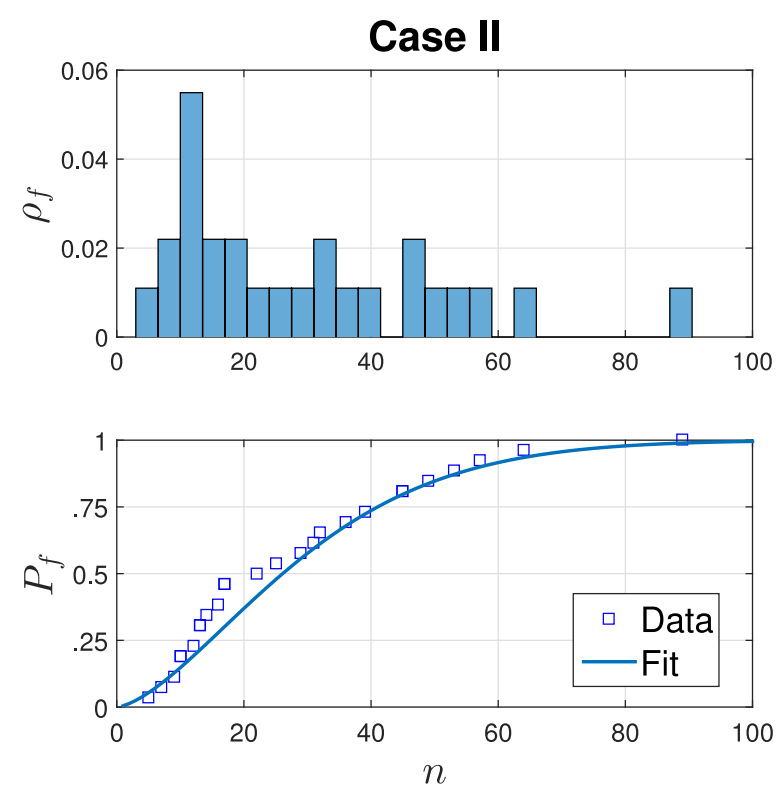

FIG. 8. Failure probability density distribution (above) and failure probability distribution (below) for case II.

There were three failure modes that occurred during mechanical testing, and examples of failed nozzles in each mode are shown in Figure 9. The most common was formation of a planar crack near the location of the highest stresses in the FEA analysis of the final design, corresponding to the plane containing the central axes of the two orifices, and is therefore called orifice failure. In the case of the nozzle without clamps, this mode sometimes also led to another planar crack which began in the sac volume on the side directly opposite from the orifices, which would cause the nozzle to split into two pieces of roughly the same size, and is called splitting failure. This mode was seen only in case I, and in roughly one third of the failures of these nozzles. This failure mode was not seen in case II, probably because the much stiffer clamps prevented the nozzle from expanding enough after orifice failure to cause

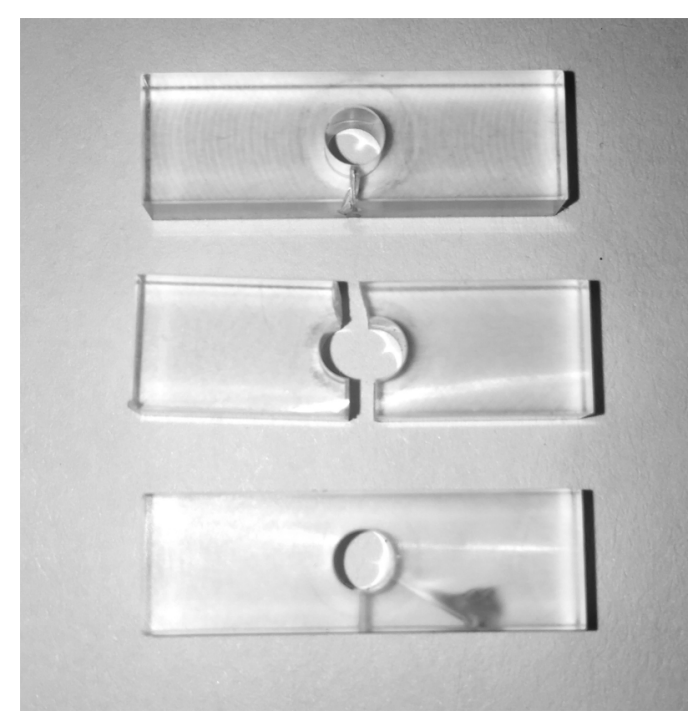

FIG. 9. A photograph of the three failure modes found during mechanical testing: orifice (top), splitting (middle), and sac volume (bottom). 


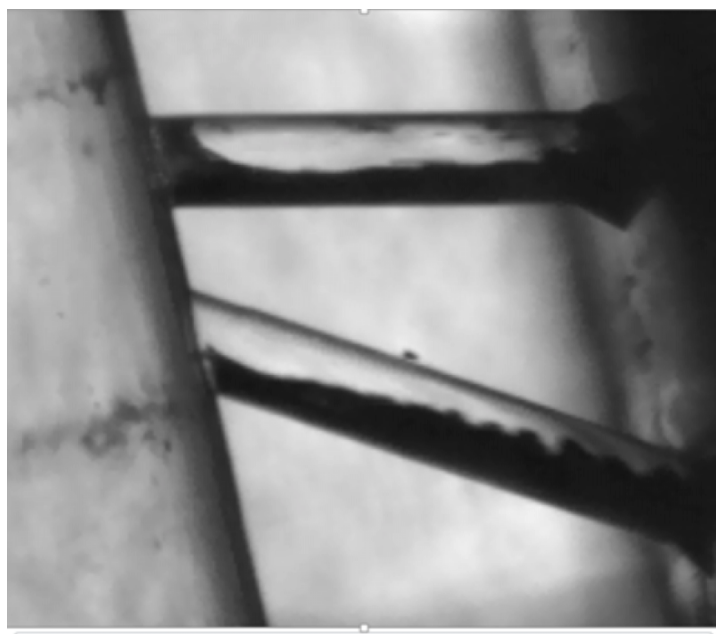

FIG. 10. Representative image of cavitation in the interior flow of the experimental OTN.

this second failure on the other side of the sac volume. The third mode involved one or more planar cracks forming in the sac volume at locations other than that seen in splitting mode, which was seen only in the cases where clamps and a small, unknown clamping force were used. This has been termed sac volume failure. This occurred in almost $50 \%$ of the failures in case II, and suggests an increased probability of failure in the sac volume as compared to the orifices.

A representative image showing the flow inside the nozzle is displayed in Figure 10. All tests were operated at atmospheric back pressure, and the orifice flow cavitated easily.

\section{DISCUSSION}

The frequently observed mechanical failure modes can be explained by the stress field in the acrylic nozzle from the FEA, which is shown in Figure 11 for case I. Similar to the simplified model, the stress is larger along the smaller dimension of the nozzle, but the nozzle has to be this thin for spray formation to be visually accessible. The orifices act as stress raisers within this already concentrated stress region, which explains the difficulty of making a durable OTN that is small enough to allow visual access to spray formation and interior flow. The largest stresses are found at the lower inlet corner of the lower orifice, which is the sharpest corner in the interior geometry, shown at the bottom of Figure 11. This is also where the nozzles failed in the orifice and splitting failure modes, which represented the majority of the mechanical failures. This was the location for the maximum tensile stress also in the case II FEA, and so the sac volume failure mode found during testing indicates that the nozzle does not always fail where the stress is greatest. This is reflected in the fact that the failure probability formulation in Equation (1) depends on the stress distribution in the entire piece.

Using the constants calculated from the mechanical testing results, the failure probability of the finalized design can be estimated for untested conditions using estimations for element stresses and volumes from FEA. The same loading as cases I and II is tested, but with the state of clamping reversed

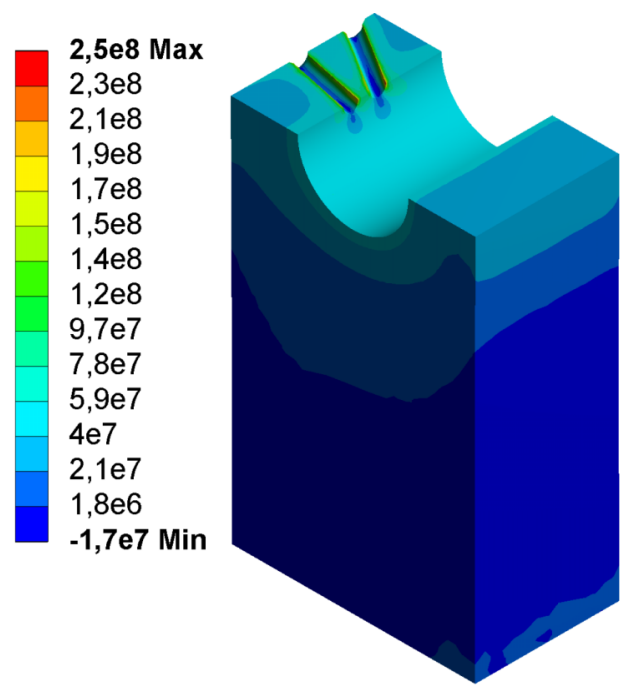

(a)

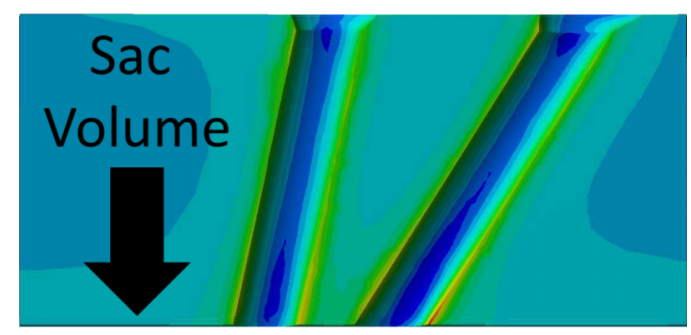

(b)

FIG. 11. (a) OTN stress distribution (Pa) in case I (b) enlarged view of stress concentration near orifices.

to examine the effect of this design feature. The results are shown with the original curve fits to the mechanical test data in Figure 12. The addition of the sapphire clamps for both cases results in a decrease in failure probability as a function of loading cycles.

The outliers in the observed experimental failure distribution for case II, which are shown in Figure 13, may be due to the accidental application of a non-negligible clamping force during assembly. To test this hypothesis, FEA was used

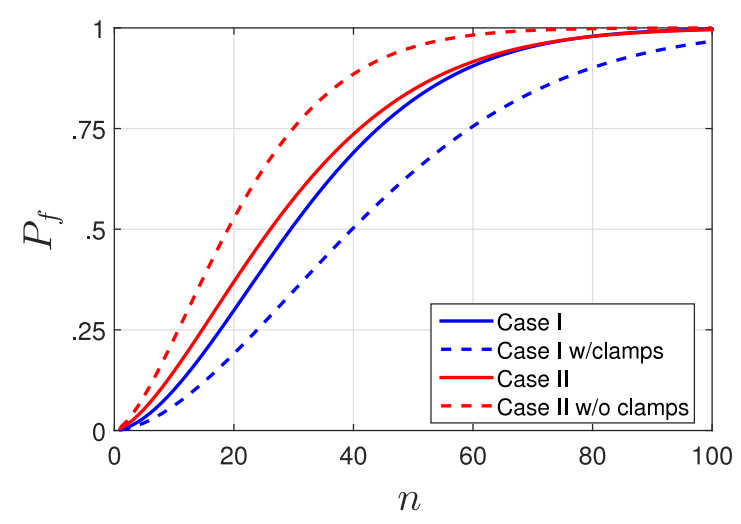

FIG. 12. Failure probability as a function of load cycle for the tested cases (solid lines) and the estimated failure probabilities calculated from FEA results (dashed lines) indicated the effect of the supporting sapphire clamps. 


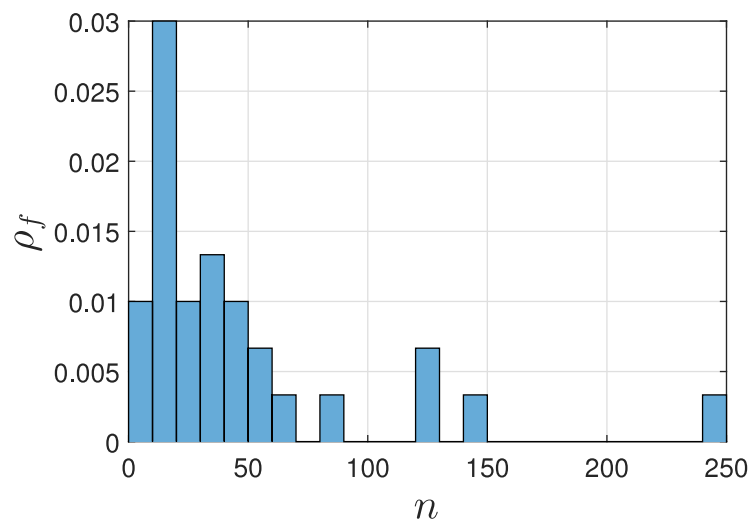

FIG. 13. Raw failure probability density distribution showing outliers with exceptional lifetimes for case II mechanical testing.

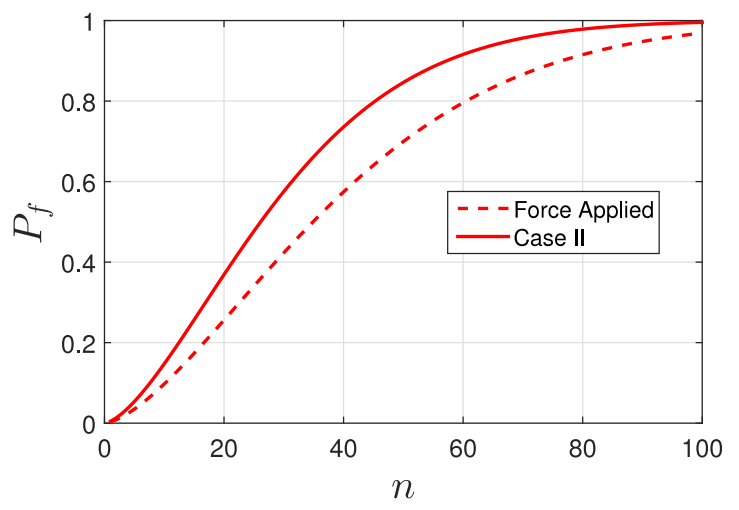

FIG. 14. Failure probability distributions for the case II data with the outliers excluded, and the estimated failure probability from FEA with $10 \mathrm{kN}$ of applied force.

to estimate the stress distribution in the nozzle with $10 \mathrm{kN}$ clamping force in each bolt. The estimated failure probability decreased when the clamping force was added, which is shown in Figure 14.

Wall cavities appear on the side of the orifices furthest away from the injector body in Figure 10, where the inlet corner is sharper. The cavities extend almost all the way to the orifice exits, a regime known as supercavitation, where they shed clouds that perhaps survive outside the injector body. No evidence of string cavitation in the sac volume can be seen, although vorticity was evident in the high-speed videos. The wrinkled cavity surface in the lower orifice indicates that turbulence in the main flow develops later in the orifice. In the upper orifice, the cavity is in the supercavitation regime on the lower side and in the cloud shedding regime on the upper side. Although it was not tested, it is likely that elevated back pressures would not affect the performance of the design, given that the injection pressure is usually orders of magnitude larger than relevant back-pressures.

\section{CONCLUSIONS}

A design for an optically transparent injector nozzle that provides access to the interior flow and spray formation at realistic injection pressures and scales has been presented.
The design process utilized FEA to provide estimates for the stress in the nozzle, which allowed trends affecting the nozzle's durability to be identified, and trends in the design's failure probability to be estimated. This design is able to reproduce and grant visual access to the actual interior and spray flows of realistic fuel injectors, while achieving an acceptable lifetime for experimental studies requiring many repetitions. This is achieved with clamps that limit the expansion of the acrylic nozzle piece, which has been shown to lower its likelihood of failure. It also seems likely that applied external force decreases the failure probability of the nozzle even further.

\section{FUTURE WORK}

Based on the results of this study, the simplest improvement to this design would be to improve the accuracy and repeatability of the applied clamping force. One possibility to improve repeatability could be to combine the clamping mechanism into a single fixture, so that the clamping force on the nozzle is applied all at once, instead of stepwise in different locations with the tightening of individual screws. A strain gauge could then be used to measure the stress at an external point on this single fixture, which would provide a quantitative indication of the force level within the assembly.

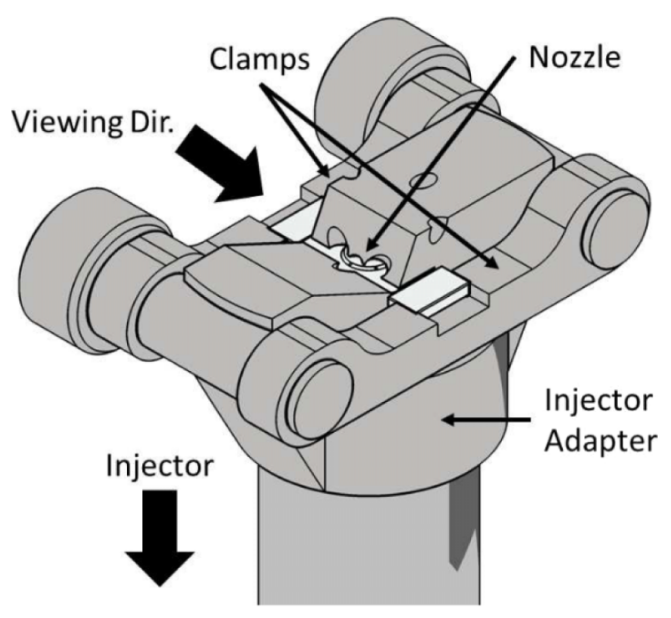

(a)

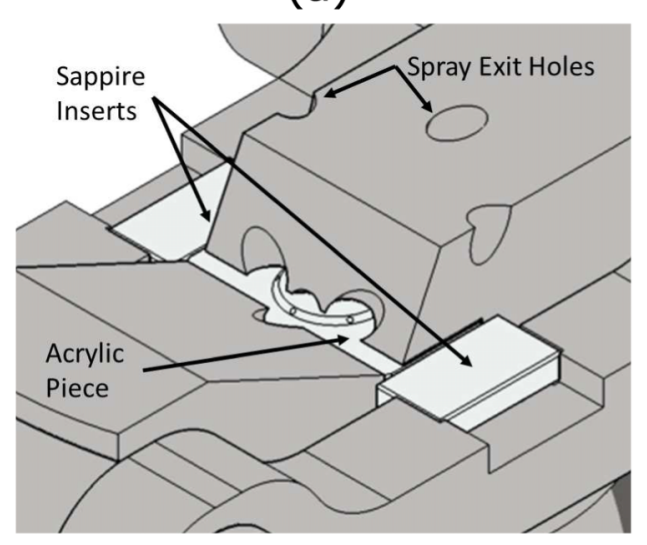

(b)

FIG. 15. (a) Illustration of design for road vehicle diesel engine injector and (b) close up of nozzle region. 


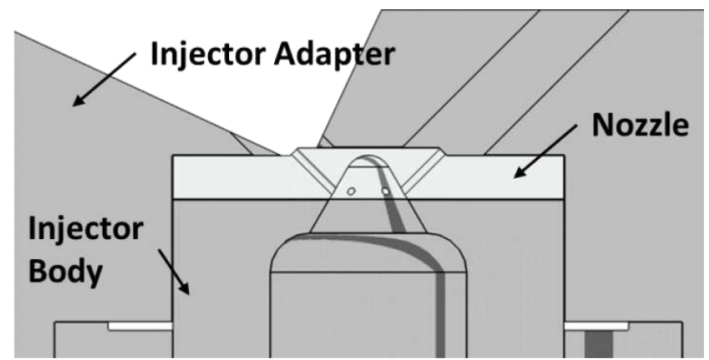

(a)
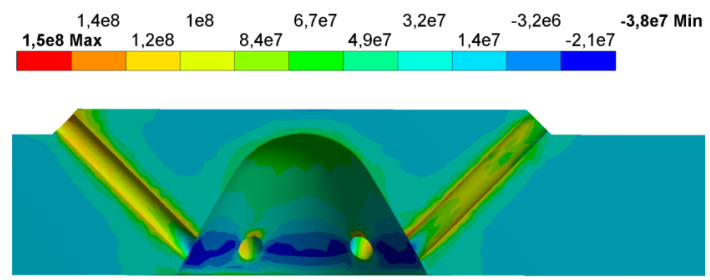

(b)

FIG. 16. (a) Illustration of cross section of road vehicle diesel engine injector and (b) FEA results showing stress distribution.

FEA could be used to optimize the location of the strain guage, as well as find an approximate relationship between the strain on the assembly surface and the clamping force on the nozzle. Relying solely on the torque levels on individual components is not recommended, because torque is related to clamping forces by the level of friction at component interfaces, which is difficult to hold constant between subsequent re-assemblies.

The importance of the relationship between interior flow and spray formation is not exclusive to marine engine injector geometries, and the same design principles used for this nozzle geometry can easily be applied to others. An interesting extension would be an optically transparent heavy duty road Diesel engine injector. An optimized design for such an injector is presented in Figure 15. A section showing the interior geometry and FEA results from using FEA results with 1500 bars injection pressure is shown in Figure 16. Based on the evidence collected in the current study, the arguments provided by the included analyses, and the peak stress in the FEA (which is lower than for the case I mechanical tests), an OTN injector with this design and orifice holes on the order of $100 \mu \mathrm{m}$ could be able to survive injection pressures of more than 1500 bars for an ensemble of injections. The performance might be greater if the clamping force is carefully controlled. With this design, the user has optical access to the entire sac volume, all orifice holes, and the formation region of 3 sprays. This injector is limited to temperatures beneath $80^{\circ} \mathrm{C}$, due to the low melting temperature of the acrylic piece. However, for the flows that are intended to be studied with this device, it is more important to match the density of the ambient outside the injector than the temperature.

\section{ACKNOWLEDGMENTS}

The authors would like to thank the EU for supporting this work under the HERCULES-C project and the Swedish Energy Agency for their support.

\section{APPENDIX: OTN DIMENSIONS}

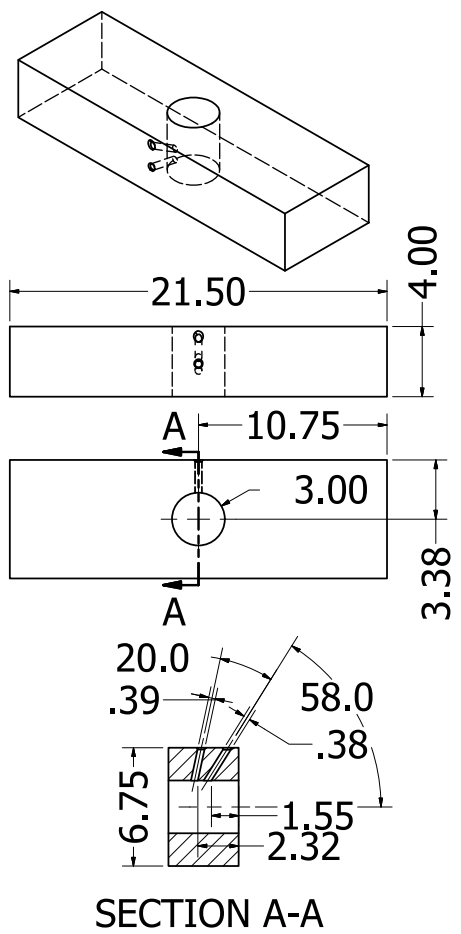

FIG. 17. Dimensions and isometric view of OTN piece. All dimensions are in $\mathrm{mm}$.

${ }^{1}$ C. Soteriou, R. Andrews, and M. Smith, SAE Technical Paper, 1486 (1999).

${ }^{2}$ M. Blessing, G. König, C. Krüger, U. Michels, and V. Schwarz, SAE Technical Paper, 1358 (2003).

${ }^{3}$ R. Payri, J. Garcia, F. Salvador, and J. Gimeno, Fuel 84, 551 (2005).

${ }^{4}$ L. Postrioti, F. Mariani, M. Battistoni, and A. Mariani, SAE Technical Paper, 2772 (2009).

${ }^{5}$ J. Desantes, R. Payri, F. Salvador, and J. Gimeno, SAE Paper, 2003.

${ }^{6}$ D. Duke, A. Swantek, Z. Tilocco, A. L. Kastengren, K. Fezzaa, K. Neroorkar, M. Moulai, C. Powell, and D. Schmidt, SAE Int. J. Engines 7, 1003 (2014).

${ }^{7}$ J. Hult, P. Simmank, S. Matlok, S. Mayer, Z. Falgout, and M. Linne, Exp. Fluids 57, 49 (2016).

${ }^{8}$ C. Arcoumanis, H. Flora, and M. Gavaises, SAE Technical Paper, 2000.

${ }^{9}$ B. A. Reid, G. K. Hargrave, C. P. Garner, and G. Wigley, Phys. Fluids 22, 031703 (2010).

${ }^{10}$ B. A. Reid, M. Gavaises, N. Mitroglou, G. K. Hargrave, C. P. Garner, E. J. Long, and R. M. McDavid, Exp. Fluids 55, 1 (2014).

${ }^{11}$ C. Badock, R. Wirth, A. Fath, and A. Leipertz, Int. J. Heat Fluid Flow 20, 538 (1999).

${ }^{12}$ N. Mitroglou, M. McLorn, M. Gavaises, C. Soteriou, and M. Winterbourne, Fuel 116, 736 (2014).

${ }^{13}$ T. Hayashi, M. Suzuki, and M. Ikemoto, Int. J. Engine Res. 14, 646 (2013).

${ }^{14}$ A. J. Butcher, P. G. Aleiferis, and D. Richardson, Int. J. Engine Res. 14, 557 (2013).

${ }^{15}$ L. A. Janosik, J. P. Gyenkenyesi, N. Nemeth, and L. Powers, NASA Technical Memorandum 106475, in 39th International Gas Turbine and Aeroengine Congress (1994).

${ }^{16}$ D. Lv, Y. Huang, Y. Tang, and H. Wang, Int. J. Adv. Des. Manuf. Technol. 67, 613 (2012).

${ }^{17}$ T. Suratwala, R. Steele, M. Feit, L. Wong, P. Miller, J. Menapace, and P. Davis, J. Non-Cryst. Solids 354, 2023 (2008).

${ }^{18}$ T. Suratwala, L. Wong, P. Miller, M. Feit, J. Menapace, R. Steele, P. Davis, and D. Walmer, J. Non-Cryst. Solids 352, 5601 (2006).

${ }^{19}$ N. Nemeth, L. Powers, J. Salem, and S. R. Choi, J. Eng. Gas Turbines Power 118, 863 (1996).

${ }^{20}$ P. P. Wang, S. Lee, and J. P. Harmon, J. Polym. Sci., Part B: Polym. Phys. 32, 1217 (1994).

${ }^{21}$ Z. Bertalan, A. Shekhawat, J. P. Sethna, and S. Zapperi, Phys. Rev. Appl. 2 , 034008 (2014); e-print arXiv:1404.4584. 